\title{
Biological effect of human serum collected before and after oral intake of Pygeum africanum on various benign prostate cell cultures
}

\author{
Stéphane Larrée ${ }^{1,2}$, Philippe Camparo ${ }^{2,3}$, Eva Comperat ${ }^{2,4}$, Delphine Boulbés ${ }^{2}$, Mohammed Haddoum ${ }^{5}$, \\ Sylvain Baulande ${ }^{6}$, Pascal Soularue ${ }^{6}$, Pierre Costa $^{7}$ and Olivier Cussenot ${ }^{2}$
}

Pygeum africanum (Tadenan) is a popular phytotherapeutic agent used in the treatment of symptomatic benign prostatic hyperplasia. The active compounds of the drug have not been identified, and determining the plasma concentration of the drug is, therefore, not possible. Because there are conflicting results on the efficacy of this drug, we aimed to investigate its effect on prostate cell growth in vitro using human serum collected before and after Pygeum africanum intake. We used primary and organotypic cultures of human prostatic stromal myofibroblast cell line WPMY and prostatic epithelial cell line PNT2. We also used fresh benign prostatic tissue. The serum of a treated man induced decreases in the proliferation of primary cells, organotypic cells and WPMY cells but not PNT2 cells. We also analysed the effect of treated serum on the gene expression profile of WPMY cells. The transcriptome analysis revealed an upregulation of genes involved in multiple tumour suppression pathways and a downregulation of genes involved in inflammation and oxidative-stress pathways. The oral intake of Pygeum africanum resulted in serum levels of active substances that were sufficient to inhibit the proliferation of cultured myofibroblasts prostatic cells. This inhibition was associated with changes in the transcriptome. Asian Journal of Andrology (2012) 14, 499-504; doi:10.1038/aja.2011.132; published online 26 December 2011

Keywords: benign prostatic hyperplasia; organotypic culture; primary culture; PNT2; Pygeum africanum; transcriptome; WPMY

\section{INTRODUCTION}

Phytotherapeutic agents have been considered the first-line treatment for benign prostatic hyperplasia (BPH) in Asia, Africa and India where they have been used for centuries. ${ }^{1}$ In Europe, Pygeum africanum (PA), which is sold commercially as Tadenan, is a popular phytotherapeutic agent that has been available for more than 30 years. ${ }^{1} \mathrm{PA}$ is extracted from the bark of the African plum tree Prunus africana, an evergreen member of the rosaceae family native to the montane regions of SubSaharan Africa and the island of Madagascar. Many clinical and basic science studies have been performed to assess the clinical efficacy of PA and have yielded conflicting results.

In animal models, PA modulates bladder contractility, has an antiinflammatory activity, decreases the production of leukotrienes and other 5-lipoxygenase metabolites, inhibits fibroblast production, affects adrenal androgens and restores the secretory activity of the prostate epithelium. ${ }^{2,3}$ More recently, the antiproliferative and antiapoptotic effects of PA were demonstrated using fresh human benign prostatic cells ${ }^{4,5}$ and the cancerous prostatic human cell lines LNCaP and PC-3. ${ }^{6}$

The results of a meta-analysis examining 18 randomized controlled trials involving more than 1500 men suggested that PA modestly improves symptoms and flow measures compared to placebos. ${ }^{1}$
However, the reviewed studies were of short duration, were small in size, used varied doses and preparations with a high placebo response and rarely reported outcomes using standardized validated measures of efficacy. Phytotherapeutic agents are, therefore, not recommended for use by the European Association of Urology guidelines in the treatment of BPH-related low urinary tract symptoms. ${ }^{7}$

Basic science studies have not been able to identify the active agents of PA, although multiple putative molecular targets have been suggested. It has been hypothesized that the effects are related to flavonoids, beta-sitosterol, campesterol, atraric acid or $\mathrm{N}$-butylbenzenesulfonamide, which are found in PA extracts. The precise mechanisms are unknown, although it has been shown in vitro that atraric acid and $\mathrm{N}$-butylbenzenesulfonamide are strong inhibitors of the androgen receptor and cell growth and could potentially be used for further drug development. ${ }^{8}$

The weakness of past randomized clinical studies, the lack of identified active agents and the absence of proof that the oral absorption of the drugs results in sufficient concentrations of active agents in the blood puts in doubt the clinical efficacy of PA.

Therefore, we aimed to test the hypothesis that there is a decrease in prostatic cell growth in response to human serum collected after PA intake. We exposed cultures of various human benign prostatic cell 
types to the serum of a man collected before (HS1) and after (HS2) oral intake of PA, and we assessed the effect of the sera on cell proliferation and on RNA expression using a whole-genome RNA array.

\section{MATERIALS AND METHODS}

\section{Serum}

The serum used for cultures was collected from one 46-year-old Caucasian man without low urinary tract symptoms or clinical $\mathrm{BPH}$, with a prostate-specific antigen level of $1.2 \mathrm{ng} \mathrm{ml}^{-1}$ and with a blood testosterone level of $8 \mathrm{nmoll}^{-1}$. Blood was collected once (HS1) after overnight fasting and again (HS2) under the same conditions after oral intake of Tadenan (Pygeum africanum extract; Laboratoire Solvay Pharma, Garches, France) at $50 \mathrm{mg}$ twice per day over a period of 5 days. Sera were frozen at $-20^{\circ} \mathrm{C}$ after the removal of blood cells by centrifugation. The sera were diluted to concentrations of $5 \%, 10 \%$ and $15 \%$, and were used in the culture medium of various cell cultures as described below. Written consent was provided after approval by the local ethical committee.

\section{Primary cell culture}

Primary cultures of prostatic myofibroblasts and fibroblasts (PMFs) were prepared using five different histologically confirmed BPH samples. The samples were collected at the time of surgery from five men who had undergone a suprapubic adenomectomy for BPH. Each sample was analysed separately. Two immortalized benign prostatic cell lines were also used: PNT2, which is of epithelial origin and was developed by our team (available in the European ECACC Collection), and WPMY, which is of myofibroblast origin (available in the American ATCC Collection).

We used the model described by Boulbès et al. ${ }^{4}$ Briefly, for each experiment, 1000 cells from each culture were seeded in a well in a 96well plate and cultured successively for $48 \mathrm{~h}$ using foetal calf serum culture medium, $24 \mathrm{~h}$ without foetal calf serum and $24 \mathrm{~h}$ with 5bromo- 2 '-deoxy-uridine. The culture medium contained human serum at a concentration of $5 \%, 10 \%$ and $15 \%$. Media were prepared with serum collected before and after oral intake of PA (HS1 or HS2). Basal proliferation was measured using cells cultured in MCDB 131 culture medium alone for PMF cells and RPMI culture medium alone for PNT2 and WPMY cells. PMF cells were used at the second passage.

The cells were then fixed and permeabilized with acid ethanol, and the DNA was denatured by heating in a formamide/trisodium citrate solution. Proliferation tests were performed using the 5-bromo-2'deoxy-uridine Labeling and Detection Kit III (Roche Diagnostics, Mannheim, Germany) according to the manufacturer's instructions. The level of 5-bromo-2'-deoxy-uridine incorporation was measured using a microtiter plate reader at $405 \mathrm{~nm}$ with a reference wavelength at $490 \mathrm{~nm}$. A nonspecific control (cells without 5-bromo-2'-deoxyuridine) was performed, and the absorbance at $405 \mathrm{~nm}$ of these cells was subtracted from each experimental value, which was subsequently reported with the corresponding negative control arbitrarily set to 1 . Luminescence was measured in a Wallac JET 1450 counter (PerkinElmer, Waltham, MA, USA) and analysed with the MicroBeta Workstation software (PerkinElmer). Experiments including controls were repeated three times for immortalized cell cultures and five times for primary cultures.

\section{Organotypic cultures}

The principle of the model of Papini et al. ${ }^{9}$ was used and adapted using an alginate culture base with 5-mm-long and 1.3-mm-wide BPH cores. We used the same $\mathrm{BPH}$ samples as were used in the primary culture cell experiments. The prostatic cores were placed in six-well plates with alginate sponges. Plates were incubated for $48 \mathrm{~h}$ with RPMI without serum and for $72 \mathrm{~h}$ with human sera collected before or after oral intake of PA (HS1 or HS2) at concentrations of 5\%, 10\% and $15 \%$.

Immunostaining was performed on $3-\mu \mathrm{m}$ sections obtained from the original alcohol formalin acetic acid fixed paraffin-embedded tissue block. Heat-mediated antigen retrieval was performed in $0.1 \mathrm{~mol}^{-1}$ citrate, $\mathrm{pH}$ 6.0, in a water bath for $30 \mathrm{~min}$. We used a Dako autostainer (Dako, Glostrup, Denmark) with a biotin blocking system, a peroxidase-label detection system and diaminobenzidine as a chromogen. The samples were incubated with antibodies against Mib-1 (M7240, 1/50; Dako) for $30 \mathrm{~min}$ at room temperature. The slides were subsequently counterstained with haematoxylin. Appropriate positive and negative controls were used. Immunoreactivity was evaluated in a quantitative way with a minimum of 200 cells counted for each specimen. Only nuclear labelling was taken into consideration, and the ratio between the number of positive cells and the total number of cells was analysed. Experiments, including controls, were repeated five times.

\section{Statistical analysis}

The proliferation indices were compared using the non-parametric Wilcoxon test. The results were considered significant when $P<0.05$.

\section{Transcriptome analysis}

The transcriptome analysis was performed using WPMY cells because PNT2 cells did not exhibit a visible change on cell growth. Cells were cultured in two 75-ml flasks. At subconfluence, the cells were grown in RPMI medium without serum for $24 \mathrm{~h}$. Next, the cells were cultured with $10 \% \mathrm{HS} 1$ or HS2 serum for $48 \mathrm{~h}$. Cells were frozen at $-80{ }^{\circ} \mathrm{C}$ before RNA was extracted according to the manufacturer's instructions (QIAamp RNA Blood Mini Kit; Qiagen, Valencia, CA, USA). The RNA was analysed using the Affymetrix HG-U133 2.0 PLUS 55 000 probe sets, and transcript expression was analysed with the MAS5 algorithm. Probe set pairs with at least one of the probe sets having a level higher than three times the surrounding noise $(3 \times 150)$ were selected. Among these pairs, a probe set from the HS2 group was considered upregulated if its level was at least twice that of the level of its paired probe set in the HS1 group. Inversely, a probe set from the HS2 group was considered downregulated if its level was at least half that of the level of its paired probe set in the HS1 group. Gene and pathway analysis was performed using Ingenuity Pathway Analysis (http://www.ingenuity.com) and a PubMed search.

\section{RESULTS}

The effect of HS1 and HS2 on WPMY myofibroblasts cells is reported in Figure 1a. There was a decrease in the mean cell proliferation indices when using the HS2 serum (1.1 vs. 1.3, $P<0.05)$. The effect of HS2 serum was apparent when using serum concentrations of $10 \%$ and $15 \%(1.2$ vs. $1.5, P<0.05)$ but not for $5 \%$ (1.01 vs. 1.04$)$.

The effect of HS1 and HS2 on PNT2 epithelial cells is reported in Figure 1b. There was no significant difference in cell proliferation between the two groups (1.15 vs. 1.13), and the overall effect of the serum was weak for all concentrations of serum used.

The effects of HS1 and HS2 on PMF cells are reported in Figure 1c. There was a significant decrease in PMF cell proliferation at all concentrations of serum in the HS2 group compared to the HS1 group (1.3 vs. 1.6, $P<0.001$ ). The effect was increased when using the $15 \%$ serum concentration (1.2 vs. 1.8).

The effects of HS1 and HS2 on organotypic cultures are reported in Figure 1d. There was a decrease in the Mib-1 antibody level when 
a

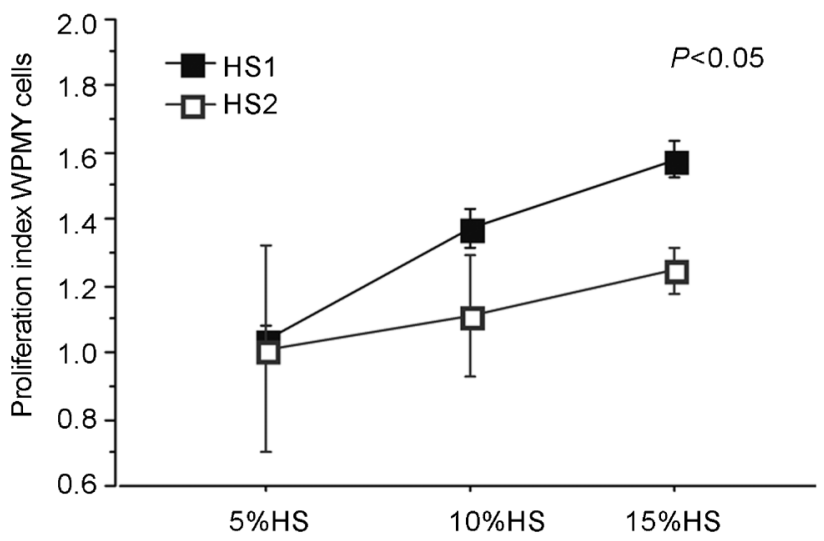

C

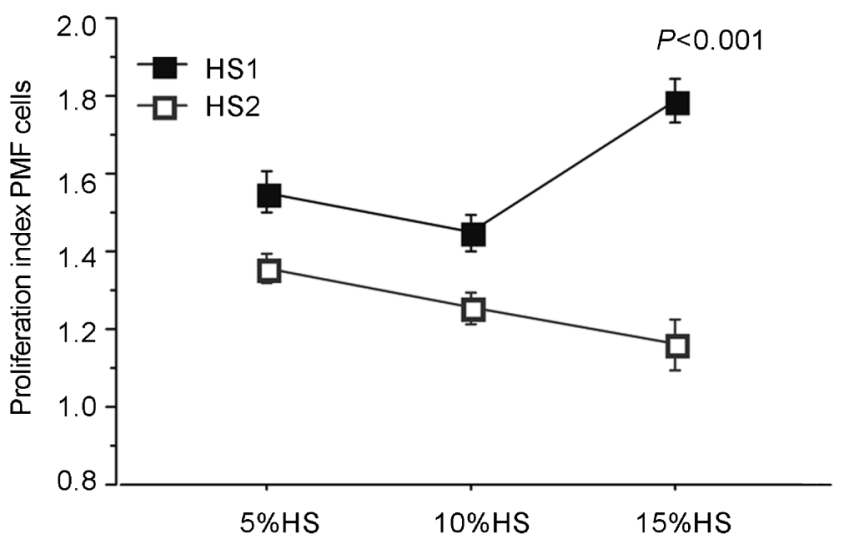

b

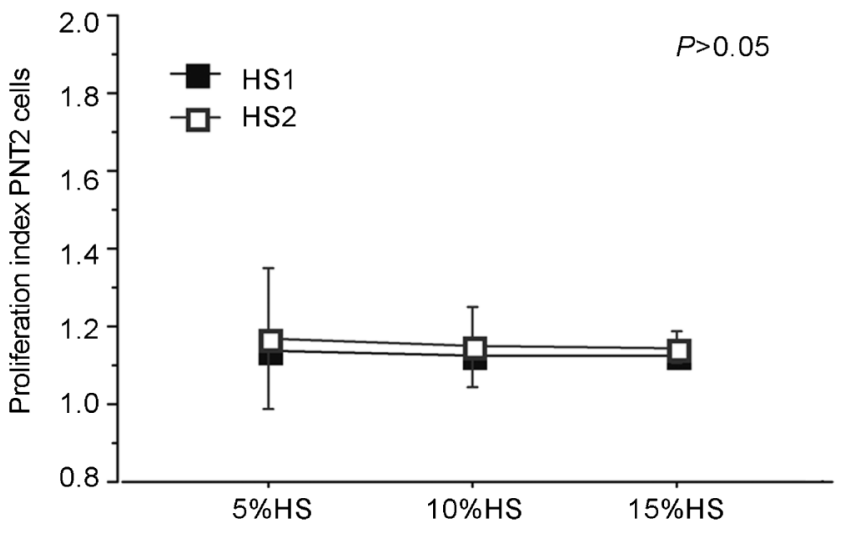

d

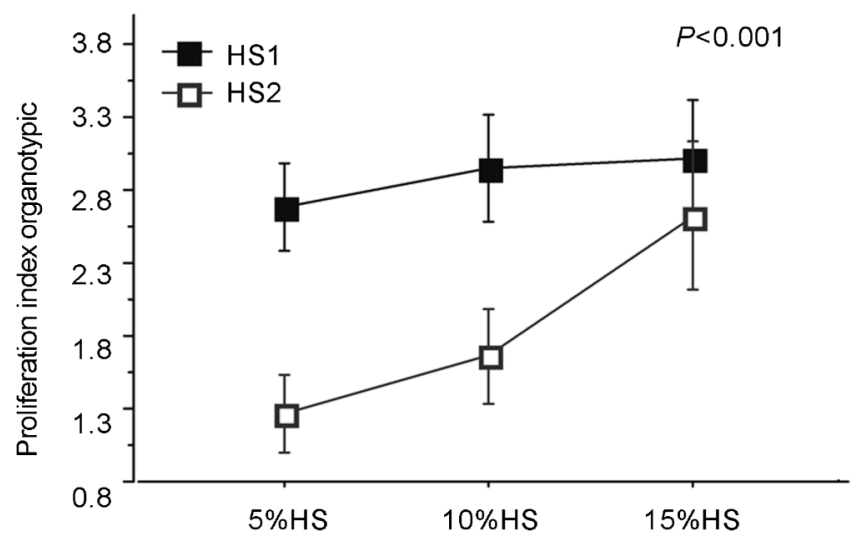

Figure 1 Dose-response effects of human sera collected before (HS1) and after (HS2) oral intake of Pygeum africanum on the proliferation of various types of prostatic cells: (a) WPMY prostatic myofibroblast cells; (b) PNT2 prostatic epithelial cell line; (c) primary fibromuscular prostatic cells (PMF); (d) organotypic cultures of fibromuscular prostatic cells. The proliferative index was the ratio of the proliferation score using human serum to the score of the control using culture media alone. PMF, prostatic myofibroblast and fibroblasts.

using HS2 at all serum concentrations (1.9 vs. $2.9, P<0.001)$. The effect decreased with increasing HS2 concentration.

The effects on the transcriptome and the probe sets with up- or downregulated activity, and their corresponding genes are reported in Table 1. A total of 25 genes were found to be differentially expressed when the serum after ingestion of PA was used. The 13 underexpressed genes were related to a specific tumour growth, fatty-acid biosynthesis, microtubule and protein transport activity, inflammatory and oxidative stress responses, signal transduction and cytoskeleton organisation. The 12 overexpressed genes were related to transcription regulation, mRNA catabolism, responses to stress, intracellular protein vesicle transport, fibroblast growth factors and other tyrosine kinase receptors (indirectly), transforming growth factor (TGF)-beta and tumour suppressor pathways.

\section{DISCUSSION}

The results obtained using various $\mathrm{BPH}$ cultures grown in the presence of serum from a man treated with PA corroborate the results previously reported for the direct application of $\mathrm{PA}$ on mouse $^{10}$ or human ${ }^{4,5}$ prostatic fibroblasts. The main criticism of PA pharmacology and clinical studies is that the precise compound responsible for the effect of $\mathrm{PA}$ is unknown and, therefore, it has never been demonstrated that the PA levels in the serum and prostate after oral absorption are as high as those used in in vitro or animal studies. To address this issue, we used the serum of a man before and after the ingested PA. This study is the first to demonstrate that the serum of a man treated with PA could induce a response in prostatic cells with relevant modification of the transcriptome and inhibition of prostatic cell growth. Nonetheless, because the experiments were performed with the serum of only one man, which is a limitation of this study, our results cannot be generalized because there may be variability in the absorption or metabolism of the drug among individuals.

The effect on prostatic cells is supported by many aspects of our study. The inhibitory effect on cell growth when using various models of prostatic cell growth reinforce the validity of these findings, especially the results obtained when using fresh prostatic cells from five different men and when using 3D organotypic cultures, which more closely resemble the in vivo conditions than the regular cell line cultures. ${ }^{4,9}$ Fresh prostatic cells in these models were more likely to 


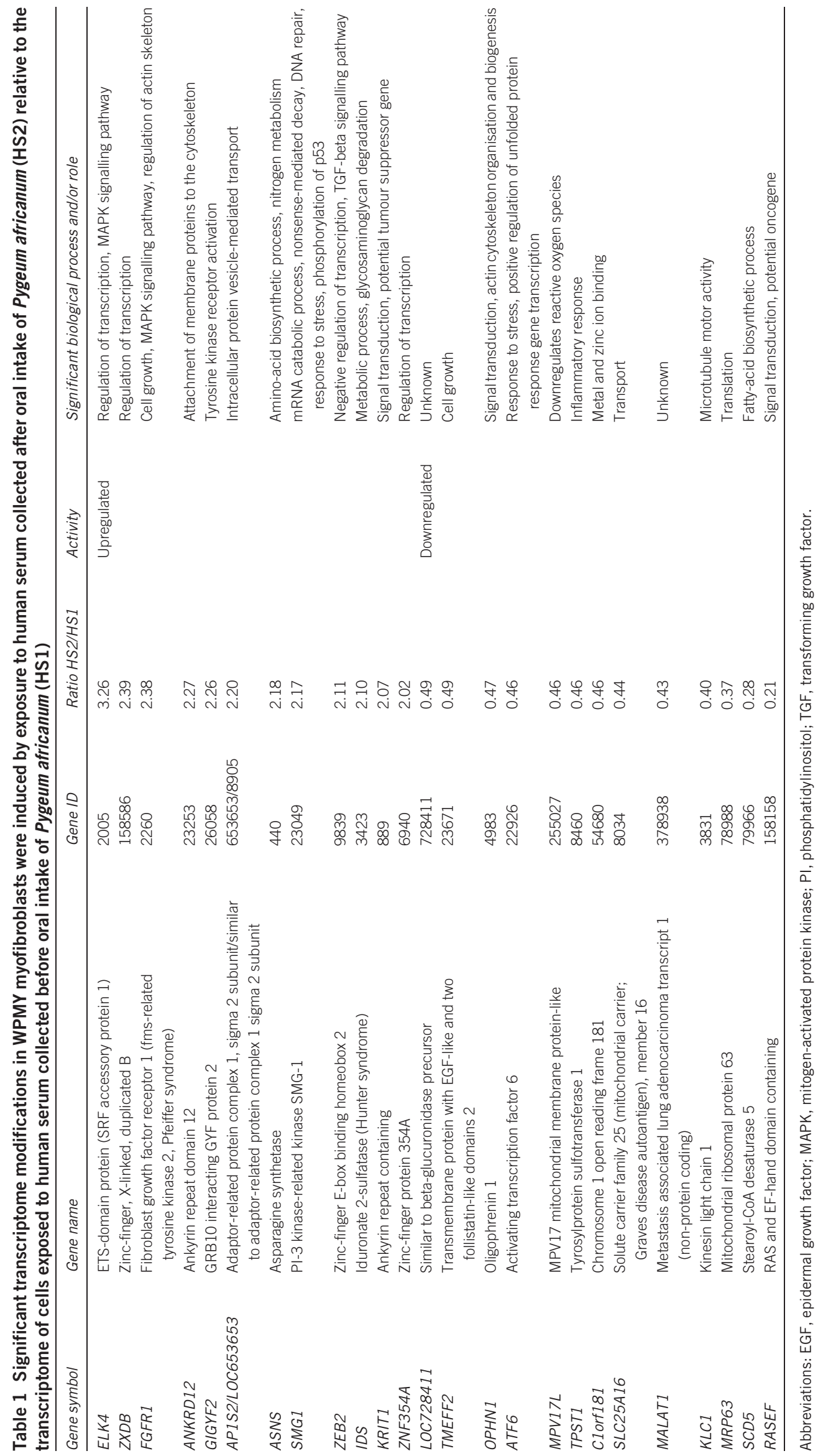


proliferate in the presence of normal serum than immortalized cells were, which is in line with earlier reports. ${ }^{4}$ Primary and organotypic cultures may, therefore, be interesting tools to analyse the effects of different drugs on prostate cell proliferation when cultured with human serum. PA did not have any observed effect on the PNT2 prostatic epithelial cells. There was little proliferative effect observed for both types of serum used; longer culture times may be necessary to see an effect. The effect on WPMY cell proliferation was observed only for the highest serum concentrations. PA was previously observed to have a similar effect on PMF and Madin-Darby canine kidney epithelial cells exposed to similar concentrations of PA. ${ }^{4}$ Therefore, it seems likely that PA is mainly active on myofibroblast growth rather than BPH epithelial cell growth, corroborating previous findings obtained using rats. ${ }^{10}$ This result may also indicate that human serum is not a suitable culture medium for prostatic epithelial cells, again highlighting the close relationship between the epithelium and the stroma during prostate growth. ${ }^{11}$

The effect of PA on cancerous cell lines has also been reported, and treatment with PA is associated with a reduced incidence of prostate cancer in TRAMP mice, ${ }^{6}$ indicating a global effect on pathological prostate growth. Our results also suggest that gene expression is modified in response to exposure to PA, supporting the hypothesis that sufficient drug levels were present in the serum to induce an effect. Additional experiments, such as measuring individual RNA and protein levels and repeating the assays using sera from multiple individuals, would be required to confirm each individual candidate. Nonetheless, this gene analysis has identified relevant candidate genes in the context of the reduction of cell growth.

The effect on prostatic cells is also supported by the transcriptome analysis because major modifications were observed in pathways previously reported to be involved in $\mathrm{BPH} .{ }^{12}$ We observed an upregulation of genes related to tumour suppressor functions (KRIT1, SMG-1) and a downregulation of genes involved in tumour progression, including genes related to inflammation and oxidative stress. It has been hypothesized that KRIT1 is a tumour suppressor gene, ${ }^{13}$ and $S M G-1$ can phosphorylate $\mathrm{p} 53$, which is a tumour suppressor gene involved in many cancers. ${ }^{14}$

Downregulation of oncogenes was also observed. RASEF is a member of the RAS oncogene family and is involved in various aspects of intracellular membrane transport, including exocytosis. ${ }^{15}$ Little is known about this gene, but it has been reported that the expression of RASEF is increased in familial cutaneous malignant melanoma, ${ }^{16}$ thereby suggesting a potential oncogenic role.

Other genes may also be linked to tumour development. MALAT-1 expression was shown to be significantly correlated with the risk of metastasis in early-stage non-small-cell lung cancer. ${ }^{17}$ Clorf181 was reported to be a breast cancer antigen. ${ }^{18}$ ASNS was found to be associated with pancreatic and gastric cancer and is associated with cell cycle progression. ${ }^{19,20} \mathrm{KLC} 1$ has been reported to be involved in kidney and breast tumours that are associated with the VHL tumour suppressor protein and in the synthesis and maintenance of primary cilia. ${ }^{21}$

Inflammatory mechanisms and responses to stress were also affected by the treated serum as illustrated by the overexpression of the SMG1 gene and the downregulation of the TPST1 and MPV17L genes, which have been shown to downregulate the production of reactive oxygen species. ${ }^{22}$ Of reactive oxygen species, $\mathrm{H}_{2} \mathrm{O}_{2}$ has been reported to be a second messenger for various physiologically active agents, such as TGF-beta and epidermal growth factor, both of which are involved in $\mathrm{BPH}$ processes.
Tyrosine kinase genes have been reported to be involved in $\mathrm{BPH}$ and prostate cancer processes. ${ }^{12}$ ZEB2, which was shown to be upregulated, plays a crucial role in the regulation of TGF-beta signalling. ${ }^{23}$ This effect may be related to $\beta$-sitosterol, which was able to significantly induce the expression and secretion of TGF-betal in primary cultures of $\mathrm{BPH}^{24}$

The overexpression of the first receptor of the fibroblast growth factor receptor family (FGFR1), a tyrosine kinase receptor, highlights the frequent impact of fibroblast growth factor family members on BPH. ${ }^{8,11,25}$ Nonetheless, the overexpression of FGFR1 in the general context of reducing proliferation is unexpected, and additional data are required for the accurate interpretation of this result because the overexpression of FGFR1 has been reported to be associated with the proliferation of both benign and cancerous prostate lesions.

Altogether, the results of this transcriptome analysis suggest the drug had an effect on prostatic cells, especially on pathways usually involved in $\mathrm{BPH} .{ }^{12}$ Nonetheless, no firm conclusion could be made regarding the different genes that were up- or downregulated because PCR was not used to confirm these results. Here again, the aim was to show that there is an effect on prostatic cells after the absorption of PA and not to elucidate the underlying mechanisms. Another aim was to describe an original method to investigate the effect of various phytotherapeutic drugs of unknown composition on prostatic cell growth that could be used to determine the underlying mechanisms.

The oral intake of PA could result in sufficient serum levels of active substances to induce a transcriptome modification and the inhibition of prostatic myofibroblast growth. Nonetheless, our results should not be construed as justifying the medical usefulness of PA but as a proof of concept that the oral absorption of PA could provide sufficient concentrations of active substances to the prostate.

\section{AUTHOR CONTRIBUTIONS}

SL performed interpretation and data analysis, and drafting of the manuscript. PC, EC and DB performed cell culture experiments. $\mathrm{MH}$ is responsible for conception of the study and data analysis. SB and PS performed transcriptome experimentation and analysis. PC made interpretation of data and critical revision of the manuscript. OC is responsible for design of the study, serum collection and critical revision of the manuscript.

\section{COMPETING FINANCIAL INTERESTS}

$\mathrm{MH}$ is working for Solvay Pharma. PC is a consultant advisor for Solvay Pharma.

\section{ACKNOWLEDGMENTS}

Solvay Pharma provided unrestricted financial support.

1 Ishani A, MacDonald R, Nelson D, Rutks I, Wilt TJ. Pygeum africanum for the treatment of patients with benign prostatic hyperplasia: a systematic review and quantitative meta-analysis. Am J Med 2000; 109: 654-64.

2 Buck AC. Is there a scientific basis for the therapeutic effects of serenoa repens in benign prostatic hyperplasia? Mechanisms of action. J Urol 2004; 172: 1792-9.

3 Levin RM, Das AK. A scientific basis for the therapeutic effects of Pygeum africanum and Serenoa repens. Urol Res 2000; 28: 201-9.

4 Boulbès D, Soustelle L, Costa P, Haddoum M, Bali JP et al. Pygeum africanum extract inhibits proliferation of human cultured prostatic fibroblasts and myofibroblasts. BJU Int 2006; 98: 1106-13.

5 Quiles MT, Arbos MA, Fraga A, de Torres IM, Reventos J et al. Antiproliferative and apoptotic effects of the herbal agent Pygeum africanum on cultured prostate stromal cells from patients with benign prostatic hyperplasia (BPH). Prostate 2010; 70 1044-53. 
6 Shenouda NS, Sakla MS, Newton LG, Besch-Williford C, Greenberg NM et al. Phytosterol Pygeum africanum regulates prostate cancer in vitro and in vivo. Endocrine 2007; 31: 72-81.

7 Madersbacher S, Alivizatos G, Nordling J, Sanz CR, Emberton M et al. EAU 2004 guidelines on assessment, therapy and follow-up of men with lower urinary tract symptoms suggestive of benign prostatic obstruction (BPH guidelines). Eur Urol 2004; 46: 547-54.

8 Roell D, Baniahmad A. The natural compounds atraric acid and $N$-butylbenzenesulfonamide as antagonists of the human androgen receptor and inhibitors of prostate cancer cell growth. Mol Cell Endocrinol 2011; 332: 1-8.

9 Papini S, Rosellini A, de Matteis A, Campani D, Selli C et al. Establishment of an organotypic in vitro culture system and its relevance to the characterization of human prostate epithelial cancer cells and their stromal interactions. Pathol Res Pract2007; 203: 209-16.

10 Yablonsky F, Nicolas V, Riffaud JP, Bellamy F. Antiproliferative effect of Pygeum africanum extract on rat prostatic fibroblasts. J Urol 1997; 157: 2381-7.

11 Ropiquet F, Berthon P, Villette JM, Le Brun G, Maitland NJ et al. Constitutive expression of FGF2/bFGF in non-tumorigenic human prostatic epithelial cells results in the acquisition of a partial neoplastic phenotype. Int J Cancer 1997; 72 : 543-7.

12 Descazeaud A, Rubin MA, Hofer M, Setlur S, Nikolaief $\mathrm{N}$ et al. BPH gene expression profile associated to prostate gland volume. Diagn Mol Pathol 2008; 17: 207-13.

13 Verlaan DJ, Davenport WJ, Stefan H, Sure U, Siegel AM et al. Cerebral cavernous malformations: mutations in Krit1. Neurology 2002; 58: 853-7.

14 Abraham RT. The ATM-related kinase, hSMG-1, bridges genome and RNA surveillance pathways. DNA Repair (Amst) 2004; 3: 919-25.

15 Shintani M, Tada M, Kobayashi T, Kajiho H, Kontani K et al. Characterization of Rab45/RASEF containing EF-hand domain and a coiled-coil motif as a selfassociating GTPase. Biochem Biophys Res Commun 2007; 357: 661-7.
16 Jonsson G, Bendahl PO, Sandberg T, Kurbasic A, Staaf J et al. Mapping of a novel ocular and cutaneous malignant melanoma susceptibility locus to chromosome 9q21.32. J Natl Cancer Inst 2005; 97: 1377-82.

17 Muller-Tidow C, Diederichs S, Thomas M, Serve H. Genome-wide screening for prognosis-predicting genes in early-stage non-small-cell lung cancer. Lung Cancer 2004; 45(Suppl 2): S145-50.

18 Scanlan MJ, Gout I, Gordon CM, Williamson B, Stockert E et al. Humoral immunity to human breast cancer: antigen definition and quantitative analysis of mRNA expression. Cancer Immun 2001; 1 : 4.

19 Takikawa M, Akiyama Y, Maruyama K, Suzuki A, Liu F et al. Proteomic analysis of a highly metastatic gastric cancer cell line using two-dimensional differential gel electrophoresis. Oncol Rep 2006; 16: 705-11.

20 Cui H, Darmanin S, Natsuisaka M, Kondo T, Asaka M et al. Enhanced expression of asparagine synthetase under glucose-deprived conditions protects pancreatic cancer cells from apoptosis induced by glucose deprivation and cisplatin. Cancer Res 2007; 67: 3345-55.

21 Lolkema MP, Mans DA, Snijckers CM, van Noort M, van Beest M et al. The von HippelLindau tumour suppressor interacts with microtubules through kinesin-2. FEBS Lett 2007; 581: 4571-6.

22 lida R, Yasuda T, Tsubota E, Takatsuka H, Matsuki T et al. Human Mpv17-like protein is localized in peroxisomes and regulates expression of antioxidant enzymes. Biochem Biophys Res Commun 2006; 344: 948-54.

23 Postigo AA. Opposing functions of ZEB proteins in the regulation of the TGFbeta/BMP signaling pathway. EMBO J 2003; 22: 2443-52.

24 Kassen A, Berges R, Senge T. Effect of beta-sitosterol on transforming growth factorbeta- 1 expression and translocation protein kinase $\mathrm{C}$ alpha in human prostate stromal cells in vitro. Eur Urol 2000; 37: 735-41.

25 Fromont G, Chene L, Latil A, Bieche I, Vidaud M et al. Molecular profiling of benign prostatic hyperplasia using a large scale real-time reverse transcriptase-polymerase chain reaction approach. J Urol 2004; 172: 1382-5. 